\title{
Tumor characteristics and metastatic sites may predict bevacizumab efficacy in the first-line treatment of metastatic colorectal cancer
}

\author{
UMUT VAROL $^{1}$, ESIN OKTAY ${ }^{2}$, MUSTAFA YILDIRIM ${ }^{3}$, ZEKI GOKHAN SURMELI ${ }^{1}$, AHMET DIRICAN ${ }^{4}$, \\ NEZIH MEYDAN ${ }^{2}$, BURCAK KARACA $^{1}$, BULENT KARABULUT ${ }^{1}$ and RUCHAN USLU ${ }^{1}$
}

\author{
${ }^{1}$ Department of Internal Medicine, Division of Medical Oncology, Faculty of Medicine, Ege University, Bornova, Izmir; \\ ${ }^{2}$ Department of Internal Medicine, Division of Medical Oncology, Faculty of Medicine, Adnan Menderes University, Aydın; \\ ${ }^{3}$ Department of Medical Oncology, Antalya Research and Training Hospital, Antalya; ${ }^{4}$ Department of Medical Oncology, \\ Izmir Ataturk Research and Training Hospital, Bornova, Izmir, Turkey
}

Received July 31, 2013; Accepted November 4, 2013

DOI: $10.3892 / \mathrm{mco} .2013 .212$

\begin{abstract}
Colorectal cancer (CRC) is among the most frequently diagnosed cancers and a major cause of cancer-related mortality worldwide. The aim of the present study was to determine whether there was an improvement in the time to disease progression (TTP) in patients with metastatic colorectal cancer (mCRC) treated with first-line bevacizumab plus chemotherapy, according to tumor characteristics and metastatic sites. Tumor characteristics and tumor burden were considered to be predictive markers of the therapeutic efficacy of bevacizumab. The medical records of 705 patients with $\mathrm{mCRC}$ were retrospectively reviewed in three oncology centers between January, 2005 and September, 2012. A total of 101 patients completed their first-line bevacizumab-containing treatment. The median TTP was 6.93 months [interquartile range $(\mathrm{IQR})=4.20-9.80$ months] in patients treated with irinotecan, 5-fluorouracil (5-FU) and bevacizumab vs. 7.42 months (IQR=6.08-10.68 months) in those treated with oxaliplatin, 5-FU and bevacizumab $(\mathrm{P}=0.589)$. When we compared patients with pulmonary metastases (median TTP, 9.9000 months) or other metastatic patients without pulmonary metastasis (median TTP, 6.9000 months), we observed a statistically significant difference $(\mathrm{P}=0.046)$. However, when the efficacy of bevacizumab was compared in terms of other tumor characteristics (tumor grade, size and lymph node involvement) and metastatic sites, the differences were not significant $(\mathrm{P}>0.05)$. We concluded that bevacizumab may be effective in all subgroups of patients with mCRC. Furthermore, bevacizumab with combination chemotherapy may be superior
\end{abstract}

Correspondence to: Dr Umut Varol, Department of Internal Medicine, Division of Medical Oncology, Faculty of Medicine, Ege University, 315100 Kazim Dirik Street, Bornova, Izmir 35100, Turkey

E-mail: varolumut@yahoo.com

Key words: bevacizumab, colorectal cancer, metastasis, tumor characteristics to combination chemotherapy only as the first-line treatment of patients with $\mathrm{mCRC}$ and pulmonary metastasis.

\section{Introduction}

Colorectal cancer (CRC) is among the most frequently diagnosed cancers and a major cause of cancer-related mortality worldwide (1). Over several decades, the standard treatment for metastatic CRC (mCRC) has been 5-fluorouracil (5-FU)-based chemotherapy, despite the lack of a major positive effect on survival. Previous phase III trials reported that the addition of irinotecan, oxaliplatin or both to 5-FU as first-line treatment, significantly improved the survival of patients with mCRC (2-4). The addition of bevacizumab and cetuximab to standard chemotherapy was shown to increase the treatment efficacy without significantly increasing the overall toxicity $(5,6)$. The prognosis of $\mathrm{mCRC}$ has improved markedly over the last 12 years, increasing from 5 months with optimal supportive care to $\sim 2$ years with combination chemotherapy plus molecular agents (7).

Bevacizumab has several proposed mechanisms of action. It inhibits tumor angiogenesis, growth and metastasis in numerous tumor models and reduces intratumoral interstitial pressure, thereby potentially promoting the delivery of cytotoxic chemotherapy. Several anti-vascular endothelial growth factor (VEGF) agents have been tested for the treatment of mCRC; however, none are currently approved. Bevacizumab was shown to be clinically beneficial in the treatment of $\mathrm{mCRC}$, as well as that of other cancers, and was approved for use in non-small-cell lung cancer, renal cell cancer and glioblastoma, all of which are highly aggressive and proliferative tumors (8). The heterogeneity in the activity of bevacizumab in different types of tumors may be primarily associated with the differences in patient and tumor characteristics, host response factors and treatment strategy (combination of drugs, regimen and surgery of metastasis) (9).

The most extesively investigated predictive markers of mCRC are mutations of the KRAS and, possibly, BRAF genes, which are associated with resistance to epidermal growth factor receptor (EGFR)-targeted therapy (7). Several studies and genetic analyses were conducted in order to identify 
a biomarker predictive of bevacizumab treatment (10-12). However, unlike the EGFR-targeted therapies, no clinical or biological factors clearly predictive of the response or resistance to bevacizumab treatment have been identified thus far (13). Furthermore, despite nearly a decade of experience with bevacizumab, its optimal use, ideal patient population and predictive biomarkers are yet to be determined.

The present study aimed to determine whether there were differences in the time to disease progression (TTP) among patients with $\mathrm{mCRC}$ who received first-line treatment including bevacizumab, in terms of tumor grade and size, lymph node involvement, disease localization and metastatic sites, and determine whether tumor characteristics and tumor burden are predictive markers of the therapeutic efficacy of bevacizumab in the treatment of $\mathrm{mCRC}$.

\section{Materials and methods}

Study population. We retrospectively reviewed the medical records of 705 patients with $\mathrm{mCRC}$ that were referred to the Medical Oncology Departments of three oncology centers: Ege University (Bornova/Izmir, Turkey), Aydin Adnam Menderes University (Aydın, Turkey) and Antalya Research and Training Hospital (Antalya, Turkey) between January, 2005 and September, 2012. Age, gender, staging, date of diagnosis, date of metastasis, localization of the primary tumor, metastatic sites, histological findings and all subsequent treatments (chemotherapy, radiation therapy and surgery) were recorded. The protocol of this retrospective study received approval from the Institutional Review Boards of the involved institutions.

Study characteristics and inclusion criteria. TTP was the investigated primary endpoint, which was defined as the time from the initiation of chemotherapy to the first documentation of disease progression. The first documentation of progressive disease (PD) was based on the definition of PD in the guidelines of the Response Evaluation Criteria in Solid Tumors (RECIST) (14), death from any cause in the absence of previously documented PD and the investigator's clinical judgment of PD. We censored the data recorded from the last clinical visit for patients who died without known PD. Only the medical records of patients who received treatment containing bevacizumab and either irinotecan or oxaliplatin were included, whereas those of patients who did not complete the first-line chemotherapy due to treatment intolerance, rejection of further treatment or drug toxicity were excluded. Patients who had undergone a metastasectomy or received previous chemotherapy for metastatic disease were also excluded, whereas those with a history of adjuvant chemotherapy were included, provided the adjuvant treatment was completed $\geq 6$ months prior to the initiation of the treatment assessed in this study.

Treatment regimens and dosage. All patients received one of the following regimens, with the addition of bevacizumab: 5-FU plus leucovorin and oxaliplatin (modified FOLFOX6), capecitabine plus oxaliplatin (XELOX), irinotecan and capecitabine (XELIRI), tegafur plus uracil (UFT)-irinotecan or leucovorin, 5-FU and irinotecan (FOLFIRI). The doses of the drugs were as follows: $100-130 \mathrm{mg} / \mathrm{m}^{2}$ oxaliplatin or $180 \mathrm{mg} / \mathrm{m}^{2}$ irinotecan; $400 \mathrm{mg} / \mathrm{m}^{2}$ leucovorin, 1,250-2,000 mg/m² capecitabine, $300 \mathrm{mg} / \mathrm{m}^{2}$ UFT, 5-FU intravenous bolus administration of $400 \mathrm{mg} / \mathrm{m}^{2}$ or 24-h infusion of $2,400 \mathrm{mg} / \mathrm{m}^{2}$ 5-FU with a chemotherapy infusion pump; and bevacizumab, $5 \mathrm{mg} / \mathrm{kg}$ every 2 weeks or $7.5 \mathrm{mg} / \mathrm{kg}$ every 3 weeks. In each arm of the 5-FU-based regimens (oral or infusional), the doses of irinotecan, oxaliplatin or bevacizumab were comparable.

Tumor assessment. Baseline tumor assessment was performed in all the patients via abdominopelvic computed tomography (CT), magnetic resonance imaging and chest CT to exclude additional metastases. Assessments were performed every 6-8 weeks until PD or discontinuation of chemotherapy. Patients with responsive or stable disease at the time of treatment withdrawal were followed up every 6-8 weeks until PD. The patients also received scans every $6-8$ weeks following cessation of chemotherapy and the tumor response was assessed based on the RECIST guidelines (14).

Statistical analysis. SPSS software, version 11.0 (SPSS Inc., Chicago, IL, USA) for Windows was used for statistical analysis. The primary efficacy analysis compared TTP among patients treated with bevacizumab-based regimens. Descriptive data are expressed as frequency or central tendency measures. We calculated the median follow-up time in order to describe TTP. The median TTP values for patient subgroups were estimated and compared using the Mann-Whitney U or Kruskal-Wallis tests. The survival probability was calculated using the product limit method of Kaplan Meier. The differences in survival between groups were determined using the log-rank test. $\mathrm{P}<0.05$ was considered to indicate a statistically significant difference. All available variables are presented as number and percentage and the results are expressed as the means \pm SD.

\section{Results}

Patient characteristics. Among the 705 patients with mCRC who were treated at the aforementioned oncology centers, 101 patients who had completed their first-line bevacizumab-containing chemotherapy were included in this study, with $60.4 \%$ of the patients being male and $39.6 \%$ female. The median age of the patients was 56 years ( $\mathrm{IQR}=49.5-64.0$ years), $33(32.7 \%)$ patients had primary rectal cancer and $68(67.3 \%)$ had primary colon cancer. Approximately half of the patients had T3 disease $(n=45,44.6 \%)$, whereas only 2 patients had T2 disease. Overall, $50.5 \%$ of the tumors were moderately differentiated, $14.9 \%$ were poorly differentiated and $7.9 \%$ were well-differentiated, whereas in $26.7 \%$ of the cases the data with regard to the grade was missing. The tumor characteristics are summarized in Table I.

Comparison of TTP among treatment regimens. Among the selected 101 patients, 27 had previously received adjuvant therapy and had developed metastasis during follow-up. The chemotherapy regimens administered in the present study included irinotecan $(n=59,58.4 \%)$ and oxaliplatin $(n=42,41.6 \%)$, together with an infusion of 5-FU $(n=64,63.4 \%)$ or oral 5-FU regimens $(n=37,36.6 \%)$. The median TTP was 6.93 months (IQR=4.20-9.80 months) in patients treated with irinotecan,5-FU and bevacizumab, vs. 7.42 months (IQR=6.08-10.68 months) in those treated with oxaliplatin, 5-FU and bevacizumab. 
Table I. Tumor characteristics.

\begin{tabular}{|c|c|c|c|c|}
\hline Tumor characteristics & No. & Percentage & Valid (\%) & Cumulative (\%) \\
\hline \multicolumn{5}{|l|}{ Localization } \\
\hline Colon & 68 & 67.3 & 67.3 & 67.3 \\
\hline Rectum & 33 & 32.7 & 32.7 & 100.0 \\
\hline \multicolumn{5}{|l|}{ Depth of invasion } \\
\hline Undefined & 35 & 34.6 & 34.7 & 34.7 \\
\hline $\mathrm{T} 2$ & 2 & 2.0 & 2.0 & 36.6 \\
\hline $\mathrm{T} 3$ & 45 & 44.6 & 44.6 & 81.2 \\
\hline $\mathrm{T} 4$ & 19 & 18.8 & 18.8 & 100.0 \\
\hline \multicolumn{5}{|l|}{ Histology } \\
\hline Adenocarcinoma & 96 & 95.0 & 95.0 & 95.0 \\
\hline Mucinous adenocarcinoma & 4 & 4.0 & 4.0 & 99.0 \\
\hline Signet ring cell carcinoma & 1 & 1.0 & 1.0 & 100.0 \\
\hline \multicolumn{5}{|l|}{ Grade } \\
\hline Well-differentiated & 8 & 7.9 & 10.8 & 10.8 \\
\hline Moderately differentiated & 51 & 50.5 & 68.9 & 79.7 \\
\hline Poorly differentiated & 15 & 14.9 & 20.3 & 100.0 \\
\hline Total & 74 & 73.3 & 100.0 & \\
\hline Missing & 27 & 26.7 & & \\
\hline Total & 101 & 100.0 & & \\
\hline
\end{tabular}

Table II. Comparison of TTP among different treatment regimens.

\begin{tabular}{lccc}
\hline Regimens & No. & Median TTP (months) & IQR \\
\hline Irinotecan-based & 59 & 6.93 & $4.20-9.80$ \\
Oxaliplatin-based & 42 & 7.42 & $6.08-10.68$ \\
XELIRI/UFT-irinotecan + bevacizumab & 11 & 9.77 & $6.50-15.37$ \\
FOLFIRI + bevacizumab & 48 & 6.83 & $4.15-9.63$ \\
XELOX + bevacizumab & 26 & 7.88 & $6.03-13.70$ \\
FOLFOX + bevacizumab & 16 & 6.95 & $6.05-8.66$ \\
\hline
\end{tabular}

TTP, time to disease progression; IQR, interquartile range; XELIRI, irinotecan and capecitabin; UFT, tegafur plus uracil; FOLFIRI, 5-fluorouracil (5-FU) and irinotecan; XELOX, capecitabine plus oxaliplatin; FOLFOX, 5-FU plus leucovorin and oxaliplatin.

Although the TTP in patients who received oxaliplatin, 5-FU and bevacizumab was longer compared with that in patients who received irinotecan, 5-FU and bevacizumab, the difference was not statistically significant $(\mathrm{P}=0.589)$. The median TTP in the patients who received oxaliplatin-based regimens (XELOX and FOLFOX) was 7.88 and 6.95 months (IQR=6.03-13.70 and 6.05-8.66 months), respectively, vs. 9.77 and 6.83 months (IQR=6.50-15.37 and 4.15-9.63 months), in those who received irinotecan-based regimens (XELIRI or UFT-irinotecan, respectively). However, the difference in TTP between the patients that received oxaliplatin and irinotecan was not statistically significant ( $\mathrm{P}=0.131$ and 0.204 , respectively). A comparison of the TTP among treatment regimens is shown in Table II.

Localization of metastases. A total of 17 patients developed pulmonary metastases, 60 had hepatic metastases, 8 had pulmonary and hepatic metastases and 16 developed metastases other than pulmonary or hepatic (bone, peritoneal, lymphoid, adrenal, ovarian or a combination of the above). First, we performed four-group comparisons in terms of TTP and observed no statistically significant difference $(\mathrm{P}=0.134)$. Subsequently, we compared the four groups with each other and observed a statistically significant difference only between patients with and those without pulmonary metastases $(\mathrm{P}=0.046)$. We subsequently performed a subgroup analysis and TTP was found to be significantly longer in patients with pulmonary metastasis only (median TTP, 9.9000 months) compared to that in patients with metastases in other organs, without pulmonary or hepatic involvement (median TTP, 6.6667 months) $(\mathrm{P}=0.022)$. Similar differences in TTP were observed between patients with any pulmonary metastasis (pulmonary only, pulmonary and hepatic, or pulmonary and other organ involvement) (median TTP, 9.7667 months) and those with any metastasis, without pulmonary or hepatic involvement (median TTP, 6.6667 months) $(\mathrm{P}=0.035)$ (Table III). The differences in the median TTP between patients with tumors that were well (7.26 months), moderately (7.06 months) and poorly differentiated (8.8 months) were not statistically significant $(\mathrm{P}=0.92)$. Furthermore, the efficacy of bevacizumab was assessed according to other tumor characteristics, such as tumor size and lymph node involvement, 
Table III. Efficacy of bevacizumab-based treatment according to the localization of metastases.

\begin{tabular}{|c|c|c|c|c|}
\hline Metastases & No. & Median TTP (months) & IQR & P-value \\
\hline Pulmonary & 17 & 9.9000 & $6.36-15.75$ & 0.091 \\
\hline Hepatic & 60 & 6.9667 & $4.81-10.61$ & \\
\hline Pulmonary & 17 & 9.9000 & $6.36-15.75$ & 0.162 \\
\hline Pulmonary + hepatic & 8 & 7.1667 & $6.38-10.49$ & \\
\hline Pulmonary & 17 & 9.9000 & $6.36-15.75$ & 0.022 \\
\hline Other organs ${ }^{\mathrm{b}}$ & 16 & 6.6667 & $3.92-8.73$ & \\
\hline Hepatic & 60 & 6.9667 & $4.81-10.61$ & 0.939 \\
\hline Pulmonary + hepatic & 8 & 7.1667 & $6.38-10.49$ & \\
\hline Hepatic & 60 & 6.9667 & $4.81-10.61$ & 0.257 \\
\hline Other organs ${ }^{b}$ & 16 & 6.6667 & $3.92-8.73$ & \\
\hline Pulmonary + hepatic & 8 & 7.1667 & $6.38-10.49$ & 0.342 \\
\hline Other organs ${ }^{b}$ & 16 & 6.6667 & $3.92-8.73$ & \\
\hline Any pulmonary ${ }^{\mathrm{a}}$ & 25 & 9.7667 & $6.40-13.75$ & 0.035 \\
\hline Other organs ${ }^{\mathrm{b}}$ & 16 & 6.6667 & $3.92-8.73$ & \\
\hline
\end{tabular}

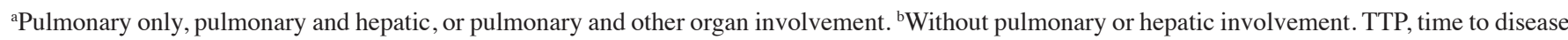
progression; IQR, interquartile range. Bold print denotes statistical significance.

and tumor localization; however the differences were not statistically significant $(\mathrm{P}=0.30,0.81$ and 0.6 , respectively).

Any additional treatments administered following first-line therapy were not specified by this analysis, as they could not be standardized. A high proportion of the patients $(85 \%)$ who received a first-line oxaliplatin-based treatment received second-line irinotecan regimens and $84 \%$ of the patients who received first-line irinotecan-based treatment received oxaliplatin regimens as a second-line therapy. Of those patients, $33 \%$ were subsequently treated with cetuximab or continued with bevacizumab together with another chemotherapy regimen following first-line treatment. Only one patient was treated with a single-agent 5-FU regimen as the second-line treatment.

\section{Discussion}

The findings reported by a number of well-designed clinical trials have resulted in significant advances in the management of mCRC $(5,7)$. These iterative studies fostered the evolution from a standard, single-agent approach using 5-FU to novel combination regimens including irinotecan and oxaliplatin. The integration of oxaliplatin and irinotecan in the management of patients with advanced-stage disease has significantly prolonged the median survival time $(2,3)$ and the administration of molecular agents, such as bevacizumab and cetuximab, in addition to chemotherapeutic modalities, has achieved an improvement in disease response rates, as well as other significant improvements, with minimal treatment-related toxicity $(5,6)$.

Angiogenesis and VEGF play a pivotal role in cancer neoangiogenesis and their involvement in CRC growth and development has been extensively investigated (15). VEGF is expressed early in the progression of CRC and its level of expression is strongly correlated with invasiveness, vascular density, metastasis, recurrence and poor prognosis $(16,17)$. Angiogenesis is a critical step in the metastatic cascade of CRC and the development of anti-angiogenic approaches, particularly anti-VEGF therapy, was shown to be promising in the treatment of patients with metastases, particularly those arising from CRC and, to a lesser degree, neuroendocrine carcinoma (18). Bevacizumab is a humanized immunoglobulin G1 monoclonal antibody that selectively binds to and neutralizes the biological activity of human VEGF (13). The neutralization of the biological activity of VEGF results in inhibition of the outgrowth of new blood vessels and a subsequent reduction in tumor growth. Bevacizumab acts synergistically with chemotherapy in colorectal, lung and renal cell carcinomas $(19,20)$. Additionally, the combination of bevacizumab and irinotecan was shown to be effective against recurrent high-grade glioblastoma multiforme (21).

Treatment with bevacizumab was shown to be effective in improving the clinical outcome of a specific group of patients. Numerous markers, including CD133 (11), circulating tumor cell count (12), lactate dehydrogenase level (22), carbonic anhydrase 9 (23), CA 125 (24), CA 19.9 (25), visceral fat area (26), SHMT1 polymorphism (27), VEGF gene polymorphisms and baseline VEGF circulating level $(13,28,29)$, were reported as potential predictors of angiogenesis blockade. Although the biology of tumor angiogenesis is complex, there is a biological rationale to support such observations. However, due to the lack of randomized study design, the absence of prospective validation of data or inability to routinely use these markers, those findings were interpreted with caution and have not been used in clinical practice.

In the present study, the combination of irinotecan and bevacizumab with oral or intravenous 5-FU administration was not more efficacious compared to oxaliplatin and bevacizumab-based regimens as the first-line treatment of mCRC, which was in agreement with previously reported findings (30). In addition, there was no difference in TTP according to tumor grade, tumor localization, depth of invasion or lymph node positivity, which indicated that bevacizumab was effective regardless of tumor grade and localization. Furthermore, the 
present findings demonstrated that TTP was significantly longer in mCRC patients with pulmonary metastasis only or with pulmonary plus other organ metastasis following the addition of bevacizumab to standard chemotherapy, compared with that in mCRC patients with metastases other than pulmonary or hepatic. Therefore, pulmonary metastasis may be a factor favouring the inclusion of bevacizumab in the first-line treatment of mCRC. However, further comprehensive and randomized trials are required in order to determine a correct statement. Therefore, we concluded that bevacizumab appears to be effective in the treatment of all the tumor subgroups of patients with mCRC.

There was no significant difference regarding TTP between patients with hepatic and those with pulmonary metastasis $(\mathrm{P}=0.091)$, which may be attributed to the limited patient sample and may be considered as a significant limitation of the present study. Furthermore, the lack of differences in tumor markers among patients and the absence of an overall survival analysis were due to missing follow-up data from the patients' records.

Although the addition of bevacizumab to cytotoxic chemotherapy as first- and second-line treatment was associated with an improvement in the outcome in all the tumor subgroups of patients with $\mathrm{mCRC}$, the identification of predictive markers of anti-angiogenic therapy is of considerable interest (31). Determining whether pulmonary metastasis is of predictive value in patients with mCRC treated with regimens containing bevacizumab requires additional investigation. Therefore, future trials must focus on elucidating the mechanisms through which bevacizumab enhances the efficacy of chemotherapy, in order to identify predictive markers that may help determine the patient groups that will benefit the most from such treatment.

\section{References}

1. Parkin DM, Bray F, Ferlay J and Pisani P: Global cancer statistics 2002. CA Cancer J Clin 55: 74-108, 2005.

2. de Gramont A, Figer A, Seymour M, et al: Leucovorin and fluorouracil with or without oxaliplatin as first-line treatment in advanced colorectal cancer. J Clin Oncol 18: 2938-2947, 2000.

3. Fuchs CS, Marshall J, Mitchell E, et al: Randomized, controlled trial of irinotecan plus infusional, bolus, or oral fluoropyrimidines in first-line treatment of metastatic colorectal cancer: updated results from the BICC-C study. J Clin Oncol 25: 4779-4786, 2007.

4. Goldberg RM, Sargent DJ, Morton RF, et al: A randomized controlled trial of fluorouracil plus leucovorin, irinotecan, and oxaliplatin combinations in patients with previously untreated metastatic colorectal cancer. J Clin Oncol 22: 23-30, 2004.

5. Chaudhury P, Hassanain M, Bouganim N, et al: Perioperative chemotherapy with bevacizumab and liver resection for colorectal cancer liver metastasis. HPB (Oxford) 12: 37-42, 2010.

6. Sobrero AF, Maurel J, Fehrenbacher L, et al: EPIC: phase III trial of cetuximab plus irinotecan after fluoropyrimidine and oxaliplatin failure in patients with metastatic colorectal cancer. J Clin Oncol 26: 2311-2319, 2008.

7. Zinser-Sierra JW, Rodríguez-Ramírez S, Villalobos-Valencia R and Ramírez-Márquez M: Use of bevacizumab in metastatic colorectal cancer: report from the Mexican opinion and analysis forum on colorectal cancer treatment with bevacizumab (September 2009). Drugs R D 11: 101-111, 2011.

8. Strickler JH and Hurwitz HI: Bevacizumab-based therapies in the first-line treatment of metastatic colorectal cancer. Oncologist 17: 513-524, 2012.

9. Chibaudel B, Tournigand $\mathrm{C}$, André $\mathrm{T}$ and de Gramont A: Therapeutic strategy in unresectable metastatic colorectal cancer. Ther Adv Med Oncol 4: 75-89, 2012.

10. De Stefano A, Carlomagno C, Pepe S,et al: Bevacizumab-related arterial hypertension as a predictive marker in metastatic colorectal cancer patients. Cancer Chemother Pharmacol 68: 1207-1213, 2011.
11. Pohl A, El-Khoueiry A, Yang D, et al: Pharmacogenetic profiling of CD133 is associated with response rate (RR) and progression-free survival (PFS) in patients with metastatic colorectal cancer (mCRC), treated with bevacizumab-based chemotherapy. Pharmacogenomics J 13: 173-180, 2013.

12. Sastre J, Maestro ML, Gómez-España A, et al: Circulating tumor cell count is a prognostic factor in metastatic colorectal cancer patients receiving first-line chemotherapy plus bevacizumab: a Spanish Cooperative Group for the Treatment of Digestive Tumors study. Oncologist 17: 947-955, 2012.

13. Formica V, Palmirotta R, Del Monte G, et al: Predictive value of VEGF gene polymorphisms for metastatic colorectal cancer patients receiving first-line treatment including fluorouracil, irinotecan, and bevacizumab. Int J Colorectal Dis 26: 143-151, 2011.

14. Eisenhauer EA, Therasse P, Bogaerts J, et al: New response evaluation criteria in solid tumours: revised RECIST guideline (version 1.1). Eur J Cancer 45: 228-247, 2009.

15. Gervaz P, Scholl B, Mainguene C, et al: Angiogenesis of liver metastases: role of sinusoidal endothelial cells. Dis Colon Rectum 43: 980-986, 2000.

16. Kabbinavar F, Hurwitz HI, Fehrenbacher L, et al: Phase II, randomized trial comparing bevacizumab plus fluorouracil (FU)/leucovorin (LV) with FU/LV alone in patients with metastatic colorectal cancer. J Clin Oncol 21: 60-65, 2003.

17. Des Guetz G, Uzzan B, Nicolas P, et al: Microvessel density and VEGF expression are prognostic factors in colorectal cancer. Meta-analysis of the literature. Br J Cancer 94: 1823-1832, 2006.

18. Degirmenci M, Karaca B, Gorumlu G, et al: Efficacy and safety of bevacizumab plus capecitabine and irinotecan regimen for metastatic colorectal cancer. Med Oncol 27: 585-591, 2010.

19. Hurwitz H, Fehrenbacher L, Novotny W, et al: Bevacizumab plus irinotecan, fluorouracil, and leucovorin for metastatic colorectal cancer. N Engl J Med 350: 2335-2342, 2004.

20. Sandler A, Gray R, Perry MC, et al: Paclitaxel-carboplatin alone or with bevacizumab for non-small-cell lung cancer. N Engl J Med 355: 2542-2550, 2006.

21. Vredenburgh JJ, Desjardins A, Herndon JE II, et al: Bevacizumab plus irinotecan in recurrent glioblastoma multiforme. J Clin Oncol 25: 4722-4729, 2007

22. Scartozzi M, Giampieri R, Maccaroni E, et al: Pre-treatment lactate dehydrogenase levels as predictor of efficacy of first-line bevacizumab-based therapy in metastatic colorectal cancer patients. Br J Cancer 106: 799-804, 2012.

23. Hong YS, Cho HJ, Kim SY, et al: Carbonic anhydrase 9 is a predictive marker of survival benefit from lower dose of bevacizumab in patients with previously treated metastatic colorectal cancer. BMC Cancer 9: 246, 2009.

24. Suenaga M, Matsusaka S, Ueno M, et al: Predictors of the efficacy of FOLFIRI plus bevacizumab as second-line treatment in metastatic colorectal cancer patients. Surg Today 41: 1067-1074, 2011.

25. Formica V, Massara MC, Portarena I, et al: Role of CA19.9 in predicting bevacizumab efficacy for metastatic colorectal cancer patients. Cancer Biomark 5: 167-175, 2009.

26. Guiu B, Petit JM, Bonnetain F, et al: Visceral fat area is an independent predictive biomarker of outcome after first-line bevacizumab-based treatment in metastatic colorectal cancer. Gut 59: 341-347, 2010.

27. Budai B, Komlósi V, Adleff V, et al: Impact of SHMT1 polymorphism on the clinical outcome of patients with metastatic colorectal cancer treated with first-line FOLFIRI+bevacizumab. Pharmacogenet Genomics 22: 69-72, 2012.

28. Abajo A, Rodriguez J, Bitarte N, et al: Dose-finding study and pharmacogenomic analysis of fixed-rate infusion of gemcitabine, irinotecan and bevacizumab in pretreated metastatic colorectal cancer patients. Br J Cancer 103: 1529-1535, 2010.

29. Hansen TF, Christensen Rd, Andersen RF, et al: The predictive value of single nucleotide polymorphisms in the VEGF system to the efficacy of first-line treatment with bevacizumab plus chemotherapy in patients with metastatic colorectal cancer: results from the Nordic ACT trial. Int J Colorectal Dis 27: 715-720, 2012.

30. Colucci G, Gebbia V, Paoletti G, et al; Gruppo Oncologico Dell'Italia Meridionale: Phase III randomized trial of FOLFIRI versus FOLFOX4 in the treatment of advanced colorectal cancer: a multicenter study of the Gruppo Oncologico Dell'Italia Meridionale. J Clin Oncol 23: 4866-4875, 2005.

31. Abajo A, Boni V, Lopez I, et al: Identification of predictive circulating biomarkers of bevacizumab-containing regimen efficacy in pre-treated metastatic colorectal cancer patients. Br J Cancer 107: 287-290, 2012. 